\title{
Caudate Volume Mediates the Interaction between Total Sleep Time and Executive Function after Acute Exercise in Healthy Older Adults
}

\author{
Junyeon Won ${ }^{\mathrm{a}}$, Alfonso J. Alfini ${ }^{\mathrm{b}}$, Lauren R. Weiss ${ }^{\mathrm{a}, \mathrm{c}}$, Casandra C. Nyhuis ${ }^{\mathrm{b}}$, Adam P. Spira ${ }^{\mathrm{b}, \mathrm{d}, \mathrm{e}}$, \\ Daniel D. Callow ${ }^{\mathrm{a}, \mathrm{c}}$ and J. Carson Smith ${ }^{\mathrm{a}, \mathrm{c}, *}$ \\ ${ }^{a}$ Department of Kinesiology, University of Maryland, College Park, MD, USA \\ ${ }^{\mathrm{b}}$ Department of Mental Health, Johns Hopkins Bloomberg School of Public Health, Baltimore, MD \\ ${ }^{\mathrm{c}}$ Program in Neuroscience and Cognitive Science, University of Maryland, College Park, MD, USA \\ ${ }^{\mathrm{d}}$ Department of Psychiatry and Behavioral Sciences, Johns Hopkins School of Medicine, Baltimore, MD, USA \\ ${ }^{\mathrm{e}}$ Center on Aging and Health, Johns Hopkins Bloomberg School of Public Health, Baltimore, MD, USA
}

\begin{abstract}
Although both exercise and sleep are significant lifestyle factors in cognitive aging, the interaction of these two factors with respect to cognition remains to be determined. Also, little is known regarding the role of the basal ganglia (BG) in cognitive aging despite its involvement in both sleep and executive function. The primary objective of this study was to investigate the interaction between sleep and acute exercise on executive function performance, and secondarily, to assess if BG volume mediates this interaction. Thirty healthy older adults $(65.8 \pm 7.3$ years) completed 30 minutes of seated rest or moderate-intensity cycling exercise on different days. Structural MRI was used to assess the volumes of BG components including caudate, putamen, and globus pallidus shortly after the experimental conditions. Approximately 90 minutes after each condition, the Stroop task was administered to measure executive function. To examine sleep, participants wore a wrist actigraph for $8.0 \pm 3.6$ days prior to the first experimental session. Results revealed that while longer total sleep time (TST) was associated with shorter Stroop response time (RT), shorter TST was associated with longer RT after exercise, compared to rest, for both congruent $(p=0.029)$ and incongruent $(p=0.022)$ trials. Longer TST was correlated with greater caudate volume, and greater caudate volume was associated with exercise-related improvement in Stroop incongruent RT. Ultimately, we found that the association between longer sleep duration and faster processing speed after acute exercise was mediated by greater caudate volume. These findings suggest that TST is an important factor for acute exercise-induced cognitive improvements in older adults, and that our study is a first step in understanding the interactive effects of these important lifestyle factors in cognitive aging that might simultaneously be addressed to promote healthy cognitive aging. Future studies should examine the interactive effects of sleep and chronic exercise on cognitive function, and whether BG volume might also mediate this interaction.
\end{abstract}

Keywords: Sleep, acute exercise, caudate, basal ganglia, mediation, older adults, the Stroop task, executive function

\footnotetext{
${ }^{*}$ Correspondence to: J. Carson Smith, PhD, Department of Kinesiology, University of Maryland, College Park, MD, 20742,
} 


\section{INTRODUCTION}

Aging comes with substantial changes in sleep quantity and quality. In healthy older adults, these include more time spent in bed (TIB), reductions in total sleep time (TST) [1], greater difficulty initiating or maintaining sleep [2], greater nighttime wakefulness, and decreases in slow-wave and rapid eye movement sleep [3]. Sleep complaints are prevalent in the general population of older people, due to these normal age-related changes, as well as increases in medical problems, medications, circadian rhythm changes, and primary sleep disorders such as sleepdisordered breathing and restless legs syndrome [4].

Age-related sleep changes present an increased risk of accelerating neurodegenerative processes in the aging brain [5]. One structural neuroimaging study in older adults demonstrated an association between insomnia and structural deterioration in orbitofrontal gyrus and parietal gray matter (GM) [6]. Another study showed that greater actigraphyassessed sleep fragmentation was related to higher risk of developing Alzheimer's disease in older individuals [7]. Age-related sleep disturbances are not only related to structural changes and greater risk of developing neurodegenerative disease, but have also been linked to executive function deficits [8]. Impaired executive function performance on the Stroop task, Digit Span Backward test, and Trail Making Test has been observed in older adults with greater actigraphy-assessed sleep fragmentation [9]. Moreover, community-dwelling older adults with self-reported poor sleep have markedly impaired processing speed [10] and inhibitory control [11] compared to their age-matched counterparts with good sleep quality.

Independently, exercise has been a successful intervention to improve cognitive function in older adults [12]. It is also increasingly clear that an acute bout of exercise is associated with enhanced cognitive function in older adults. Kamijo et al. [13] assessed executive function performance using the Flanker task [14] in response to light- (36\% of $\left.\dot{\mathrm{VO}} 2_{\max }\right)$ and moderate-intensity $\left(57 \%\right.$ of $\left.\dot{\mathrm{VO}} 2_{\text {max }}\right)$ exercise bout, respectively. The results showed that moderate-intensity exercise was associated with shorter response time (RT) during the Flanker task compared to pre-exercise, yet this effect was not observed following light-intensity exercise. Another study examined the effects of acute exercise on executive performance using the Stroop task [15] following 30 minutes of moderate-intensity acute exercise in older adults with higher $(36 \pm 1.2 \mathrm{ml} / \mathrm{kg} / \mathrm{min})$ and lower $(23.5 \pm 2.8 \mathrm{ml} / \mathrm{kg} / \mathrm{min})$ cardiorespiratory fitness $\left(\dot{V}_{2} O_{2 \text { peak }}\right)$. Shorter RT was observed after exercise in both groups, and more fit participants showed significantly better performance overall relative to less fit individuals [16].

Despite the evidence regarding the effects of an acute bout of exercise on executive function, there is a gap in the literature regarding the possible interaction of exercise with sleep on executive function. Understanding interactions between these lifestyle factors is important given that both exercise and sleep are behaviors that impact cognitive function in older adults; thus, investigating this interaction could advance efforts to establish effective strategies to promote healthy cognitive aging. Therefore, our first aim was to assess if sleep moderates the effects of acute exercise on subsequent Stroop task performance. We predicted that longer TST would be associated with better executive function performance after acute exercise in healthy older adults.

We examined the role of the basal ganglia (BG) to further unravel the complex relationship between aging, sleep, exercise, and executive function. The BG are relevant to many aspects of age-related changes in sleep and cognition, and have been shown to respond to acute exercise. For example, the BG controls sleep and wakefulness through dopamine pathways [17], and lesions in the BG lead to sleep disturbance [18]. The BG has also been widely implicated in cognitive function through frontostriatal dopaminergic projections, which are postulated to boost signal-to-noise in these cortical circuits [19]. Thus, it is plausible to hypothesize that exercise-induced upregulation of $\mathrm{BG}$ dopaminergic neurotransmission $[20,21]$ is partly responsible for improved executive function after exercise. According to earlier cross-sectional studies, cardiorespiratory fitness predicts better executive function through BG volume. Verstynen et al. [22] measured cardiorespiratory fitness ( $\left.\dot{\mathrm{V} O} 2_{\max }\right)$, BG volume, and executive function performance (Flanker and task-switch tasks) in older adults. Their findings suggest that the volume of the caudate mediates the relationship between fitness and executive function, particularly cognitive flexibility, with higher cardiorespiratory fitness predicting greater task-switch accuracy through greater caudate volume. In another study of adolescents, greater cardiorespiratory fitness $\left(\dot{\mathrm{V}}_{2 \max }\right)$ was correlated with greater BG volume, which was also correlated with better Flanker interference performance [23]. 
Despite the diverse roles and functions of the BG, it remains to be determined whether BG volume mediates TST and executive function performance after acute exercise. Hence, the second aim of the present study was to examine whether BG volume mediates the association between sleep and exercise-derived changes in executive function. To this end, we utilized structural magnetic resonance imaging (MRI) to quantify BG volume in our sample. We hypothesized that BG volume would mediate the relationship between sleep and exercise-induced changes in cognitive function in the aging brain.

\section{METHODS}

\section{Participants}

Our study sample consisted of thirty-two active older adults (ages 55-80) who were primarily recruited from local senior centers in the Washington DC metro area. Participants underwent a pre-screening using a structured interview including items specific to sleep (e.g., regarding the presence of sleep disorders) and MRI safety (e.g., regarding the presence of metal implants, cardiac pacemaker, aneurysm clips, history of claustrophobia, and other potential safety hazards). Individuals reporting a history of problems initiating sleep, staying asleep, sleep apnea, a history of stroke, diabetes, high blood pressure, neurological disease (e.g., Parkinson's or Huntington's disease), current major depressive or anxiety disorder, substance abuse, or were taking psychoactive prescription medications were excluded. Prior to the first experimental visit, participants who were eligible attended a screening session in which they completed the STOP-BANG questionnaire to screen for probable obstructive sleep apnea [24], the 7-day physical activity recall questionnaire to evaluate physical activity energy expenditure [25], and the Mini-Mental State Exam (MMSE) to assess global cognitive function [26]. All eligible participants provided informed consent approved by the Institutional Review Board at the University of Maryland and obtained physician approval for moderate-intensity exercise. The present study was conducted according to the Helsinki Declaration of 1975.

Approximately 630 older adults were exposed to in-person solicitation of the present study, primarily through local senior fitness classes. Of the 241 individuals who responded to study recruitment and advertisement, 133 individuals completed the initial phone screening, 46 individuals were scheduled
Table 1

Demographic information for study participants

\begin{tabular}{lc}
\hline & $\begin{array}{c}\text { Total Sample }(n=30) \\
\text { Mean (SD) }\end{array}$ \\
\hline $\begin{array}{l}\text { Demographics } \\
\text { Age (years) }\end{array}$ & $65.8(7.3)$ \\
Sex $(n, \%)$ & \\
$\quad$ Male & $8(26.7 \%)$ \\
Female & $22(73.3 \%)$ \\
Race $(n, \%)$ & \\
$\quad$ White & $23(76.7 \%)$ \\
Black & $2(6.6 \%)$ \\
$\quad$ Hispanic & $2(6.6 \%)$ \\
Asian & $3(10 \%)$ \\
Education $(n, \%)$ & \\
$\quad \leq$ College & $11(36.7 \%)$ \\
$\quad \geq$ Graduate & $19(63.3 \%)$ \\
Physical Characteristics & \\
Height $(\mathrm{cm})$ & $167.2(9.0)$ \\
Weight $(\mathrm{kg})$ & $71.0(14.7)$ \\
BMI (kg/m $\left.{ }^{2}\right)$ & $25.2(4.0)$ \\
7-Day Physical Activity Recall Score & \\
kJ/kg/day & $136.9(16.8)$ \\
Cognitive Function & \\
MMSE & $29.3(1.0)$ \\
\hline Notes: SD, standard deviation &
\end{tabular}

Notes: SD, standard deviation; BMI, body mass index; 7-Day Physical Activity Recall Score, scores based on approximate number of hours spent in light, moderate, hard, and very hard activities as well as estimated $\mathrm{kJ} / \mathrm{kg} /$ day during past weekdays and weekends; MMSE, Mini-Mental Status Exam.

for experimental sessions, and 39 individuals were enrolled and completed the informed consent process. Of those enrolled, 36 participants completed the screening visit, and 32 participants completed the entire study protocol. Details about the participant recruitment process are reported in our previous study [27]. Of the 32 participants who completed the entire study protocol, 2 participant data were excluded from the final analysis due to button failure during the Stroop task. Table 1 illustrates demographic, physical, and cognitive data for all participants.

\section{Sleep measurement}

To obtain quantifiable measurement of objective sleep, we used wrist actigraphy (MotionLogger Watch, Ambulatory Monitoring Inc., NY, USA), a valid and reliable method to differentiate sleep from wakefulness based on detection of motion and rest [28]. Participants wore the actigraph on their nondominant wrist and actigraphy data were collected in 1-min epochs for at least 3 valid days prior to the first experimental session. Participants were instructed to remove the watch during activities involving water such as bathing or swimming, to press an eventmarking button on the device when attempting to 
initiate sleep and upon awakening in the morning, and to complete a sleep diary to verify the recorded data. We used 'Proportional Integral Mode (PIM)' to assess both signal intensity and acceleration, as it is regarded as an accurate index of sleep in older adults $[29,30]$. Sleep data were downloaded and analyzed using a validated scoring algorithm with Action-W2 software (Ambulatory Monitoring Inc, NY, USA) and manually scored by two independent raters. The sleep indices were determined by averaging across nights and included: total sleep time (TST; number of minutes slept while in bed); sleep efficiency (SE; percentage of time in bed asleep), and wakefulness after sleep onset (WASO; number of minutes awake after initial sleep bout). Each actogram was visually inspected for invalid records by a trained technician.

The sleep data were analyzed using the following method. In the absence of an event marker, we used their reported bed ("What time did you try to go to sleep?") and wake time ("What time was your final awakening?") from the diary to identify the beginning and end of rest intervals. If there was a 50\% change in light and activity levels within 30 minutes of the reported times, we used the diary time for setting that bed or wake time. If, however, the reported time did not meet this criteria, we used an autoscore procedure. Autoscore was determined by the settings in the program, which was identified using the ColeKripke algorithm [31]. If there was a 50\% change in light and activity within 15 minutes of autoscore start and end time, then we retained the times provided by autoscore. If autoscore did not fit the activity and light changes, we used judgment to determine start and end times for the interval. Participants' sleep data are presented in Table 2.

\section{Experimental conditions}

We used a within-subjects design where the order of the experimental conditions (exercise and rest) was counterbalanced across participants. Prior to each condition, participants were fitted with a heart rate (HR) monitor (Polar Electro, Kempele, Finland) and standardized instructions were provided regarding the use of the Borg 6-20 rating of perceived exertion (RPE) scale [32]. During the exercise condition, participants completed 30 minutes of continuous cycling on a Monark cycle ergometer (Monark 828E, Varbro, Sweden) in a room approximately 10 meters outside of the MRI scanner environment. The exercise session consisted of a 5-minute warm-up, followed by 20 minutes of moderate-intensity exercise, and then
Table 2

Sleep measurements and brain volume data for study participants

\begin{tabular}{lc}
\hline & $\begin{array}{c}\text { Total Sample }(n=30) \\
\text { Mean (SD) }\end{array}$ \\
\hline Actigraphic Sleep & \\
TIB (minutes) & $458.7(63.2)$ \\
TST (minutes) & $432.2(80.4)$ \\
SE (\%) & $91.7(5.7)$ \\
WASO (minutes) & $34.5(23.8)$ \\
Wear Nights & $8.0(3.6)$ \\
Self-reported Sleep & \\
STOP-Bang & $1.6(0.7)$ \\
Regional Brain Volume $\left(\mathrm{mm}^{3}\right)$ & \\
Caudate & $6402.9(790.7)$ \\
Putamen & $8375.3(1019.3)$ \\
Globus Pallidus & $4050.2(727.1)$ \\
Total Gray Matter & $605157.9(60508.5)$ \\
Intracranial Volume & $1478054.8(178097.9)$ \\
Regional Brain Volume/ICV & \\
Caudate & $0.0043(0.0004)$ \\
Putamen & $0.0056(0.0005)$ \\
Globus Palidus & $0.0027(0.0002)$ \\
Total Gray Matter & $0.4(0.01)$ \\
\hline Notes: SD, standard deviation; TIB, total time in bed; TST, total \\
sleep time; SE, sleep efficiency; WASO, wake after sleep onset; \\
Wear Nights, the number of days participants were wearing acti- \\
graphic watch; STOP-BANG, sleep apnea questionnaire; ICV, \\
intracranial volume.
\end{tabular}

a 5-minute cool-down. Participants were instructed to adjust their workload to meet an intensity that corresponds to an RPE of 15 (associated with the verbal anchor 'Hard') during the moderate-intensity segment of exercise. HR (beats per minute, BPM) and RPE were recorded every 5 minutes to measure exercise intensity. Throughout the session, participants could drink water ad libitum. Following the 30-minute exercise session, participants were seated for a 5-minute recovery period before walking across the hall to the MRI scanner control room. During the rest condition, participants were seated for $30 \mathrm{~min}-$ utes, during which time HR and RPE were recorded at 5-minute intervals, followed by a 5-minute recovery with water provided ad libitum. Reading, writing, technology use, and excessive talking were prevented throughout the exercise and rest sessions.

\section{Structural MRI acquisition}

Whole-brain MRI was conducted on a Siemens 3.0 Tesla MR scanner (Magnetom Trio Tim Syngo, Munich, Germany). A 32-channel head coil was used for radio frequency transmission and reception. Foam padding was positioned around the sides and top of the head to minimize head movement within the coil. A high-resolution T1-weighted anatomical 
image was acquired with a magnetization-prepared rapid acquisition with gradient echo sequence: matrix $=256$, field of view $=230 \mathrm{~mm}$, voxel size $=0.9 \times 0.9 \times 0.9 \mathrm{~mm}$, slices $=192($ sagittal plane, acquired right to left), slice thickness $=0.9 \mathrm{~mm}$, repetition time $=1900 \mathrm{~ms}$, echo time $=2.32 \mathrm{~ms}$, inversion time $=900 \mathrm{~ms}$, flip angle $=9^{\circ}$, and sequence duration $=4: 26 \mathrm{~min}$. The anatomical scan sequence began approximately 15 minutes after the completion of the experimental condition. The entire MRI scanning session concluded approximately 90 minutes from the end of the exercise and rest conditions.

\section{$B G$ component volume measurement}

First, the DICOM anatomical images were converted into 3D space using the Analysis of Functional NeuroImages (AFNI) Dimon program [33]. Second, the anatomical volumes were processed with FreeSurfer's automated volumetric reconstruction pipeline (recon-all) for cortical parcellation and subcortical segmentation [34]. All estimated reconstructions were visually inspected to detect segmentation errors. FreeSurfer-reconstructed anatomical images from both scanning sessions were averaged within each participant across condition using FreeSurfer's longitudinal stream function [35] to increase the accuracy of anatomical segmentation. Subsequently, the FreeSurfer-defined volumes of BG including the caudate, putamen, and the globus pallidus were calculated based on the averaged FreeSurfer's anatomical segmentation algorithm. We then examined the differences in volumes measured after each condition to ensure that the averaging approach was sound. We also quantified total GM volume as a control to test whether the hypothesized mediation effects were driven by the total brain volume rather than BG volume, specifically. Lastly, each participant's BG and GM volumes were expressed relative to total intracranial volume (ICV). This normalization approach was used to adjust brain volume estimates based on prior research indicating significant differences in ICV between males and females [36, 37], with males having a greater ICV [38]. Participants' BG component volume data are presented in Table 2.

\section{The Stroop task}

Approximately 90 minutes after each experimental condition, participants performed the Stroop task. A computerized version of the color-naming Stroop task was presented electronically using E-Prime 2.0 (Psychology Software Tools, Pittsburgh, PA). During the task, participants were seated in front of a computer screen with a keyboard resting on a desk and were instructed to perform the task using a modified keyboard with four ergonomic keys re-labeled to represent each color (e.g. 'B' for blue, ' $\mathrm{Y}$ ' yellow, ' $G$ ' for green, and ' $R$ ' for red). Participants were instructed to use their left and right index and middle fingers to indicate the color of the stimulus as quickly and accurately as possible. A total of 64 color-word trials consisting of 28 congruent (font color and written word refer to the same color), 28 incongruent (font color and written word conflict), and 8 neutral (written word does not refer to a color) conditions were presented for each participant, with a total duration of approximately 6.5 minutes. Two versions of the Stroop task comprising unique orders of color-word stimuli were counterbalanced across experimental visits. Before starting the test, a short (2-min) practice/familiarization trial was administered, during which 20 trials (8 congruent, 8 incongruent, and 4 neutral stimuli) were presented. During the practice session, prompts to cue the location of the relevant response keys were displayed on the bottom of screen (i.e. 'B (blue)', 'Y (yellow)', 'G (green)', 'R (red)'), which were not presented during performance of measured trials. The dependent variables from the task were congruent and incongruent trial accuracy (\% correct), congruent and incongruent RT (ms), and interference score calculated using the following equation: [(Incongruent RT - Congruent RT)/Congruent RT] ${ }^{*} 100$. Other neuropsychological tests were also administered before the Stroop task, but are not discussed in this study.

\section{Statistical analysis}

\section{Exercise manipulation check}

SPSS (version 21) was used for all statistical analyses. We compared HR, RPE, and Stroop task performance between the exercise and rest conditions, with the threshold for statistical significance defined as $\alpha=0.05$. Inspection of the Stroop performance data indicated that Stroop accuracy was not normally distributed, with a negative skew and large kurtosis; therefore, a non-parametric statistical test (Wilcoxon signed-rank) was used to compare exercise and rest conditions. There was no difference between BG volume measured after exercise and rest $(p=0.720)$. As expected, we did find a strong 
correlation between $\mathrm{BG}$ volume at each visit $(r=0.966, p<0.0001)$.

\section{Interactive effects between sleep and exercise}

Multiple linear regression was conducted for the first aim of the study to test the interaction of sleep (TST, SE, and WASO) and condition (exercise, rest) on Stroop task performance.

\section{Mediation analysis}

Mediation analysis was conducted for the second aim of the study to examine the mediating effect of BG volume on the relationship between sleep variables and exercise-induced change in executive function performance. First, all variables were assessed for deviation from normality using the Shapiro-Wilk test for normality and Q-Q plots. Next, the correlation between variables including TST, SE, WASO, and BG component volume were examined using Pearson's correlation coefficients. The difference between Stroop task performance after exercise and rest (exercise minus rest) was used as the dependent variable to represent exercise-induced changes in task performance. While age was included as a covariate, sex was not included in the correlation analysis since the raw volume-to-ICV fraction accounted for variance due to sex. Once the bivariate correlation was examined, bivariate correlations were used to examine the relationship between sleep (IV; independent variable) and the Stroop task performance (DV; dependent variable). Then, associations between the BG component volume (M; mediator) and sleep (IV), and the BG component volume (M) and Stroop performance (DV), were computed, respectively. Next, we conducted a bootstrapped mediation analysis for the correlation that meets the assumption of mediation to determine the effect of sleep (IV), indirectly through caudate volume (M), on Stroop task performance (DV). Mediation analyses were administered using the mediation analysis package [39]. With the 95\% confidence interval obtained from 5000 bootstrap resamples [40], the indirect mediation effect of BG volume on the relationship between sleep and exercise-altered changes in the Stroop test was estimated.

\section{RESULTS}

\section{Actigraphic sleep statistics and quality assurance}

Table 2 shows the mean $( \pm \mathrm{SD})$ for TST, SE, and WASO. On average, participants completed $41.18 \pm 39.24 \%$ of the event-marker button presses they were instructed to make, with 10 of the 30 participants not pressing the button at all while wearing the actigraph. Additionally, 2 participants removed the device for 1 night during the recording period while 1 participant removed the device for 4 nights. Periods in which no activity was recorded for at least 31 minutes, and that were not explained in the diary as naps, were excluded from the actogram as these may be periods of unreported watch removal. A total of 125 periods were excluded from all collected data, with $68 \%$ of all excluded periods being less than 2 hours.

\section{Exercise manipulation checks}

HR and RPE during exercise and rest conditions are reported in Table 3. The mean $\mathrm{HR} \pm \mathrm{SD}$ for

Table 3

Exercise outcome and cognitive task results data for study participants

\begin{tabular}{lccc}
\hline & \multicolumn{2}{c}{ Total Sample $(n=30)$} & Exercise Main Effect \\
\cline { 2 - 3 } & $\begin{array}{c}\text { Exercise } \\
\text { Mean (SD) }\end{array}$ & $\begin{array}{c}\text { Rest } \\
\text { Mean (SD) }\end{array}$ & $p$-value $(d)$ \\
\hline Exercise outcome & & & \\
$\quad$ Heart Rate (BPM) & $136.1(18.9)$ & $66.9(8.7)$ & $<\mathbf{0 . 0 0 0 1}(\mathbf{4 . 7 0})$ \\
RPE (Borg 6-20) & $14.3(1.2)$ & $6.1(0.3)$ & $<\mathbf{0 . 0 0 0 1}(\mathbf{9 . 3 8})$ \\
Stroop task & & & \\
$\quad$ Congruent RT (ms) & $1145.5(269.5)$ & $1124.8(304.0)$ & $0.542(0.07)$ \\
Incongruent RT (ms) & $1336.4(311.1)$ & $1317.0(297.9)$ & $0.510(0.06)$ \\
Interference score (ms) & $17.4(13.9)$ & $19.1(14.0)$ & $0.484(0.12)$ \\
Congruent Accuracy (\%) & $99.0(3.9)$ & $99.2(1.7)$ & $0.861(0.07)$ \\
Incongruent Accuracy $(\%)$ & $97.8(4.7)$ & $95.8(5.7)$ & $0.180(0.38)$ \\
\hline
\end{tabular}

Notes: $p$-values and effect sizes reflect condition differences; SD, standard deviation; BPM, beats per minute; $\mathrm{RT}$, response time; ms, millisecond; Interference score $=($ Incongruent RT - Congruent RT $) /$ Congruent RT $* 100 ; d$, Cohen's $d$; Bold indicates $p<0.05$. 

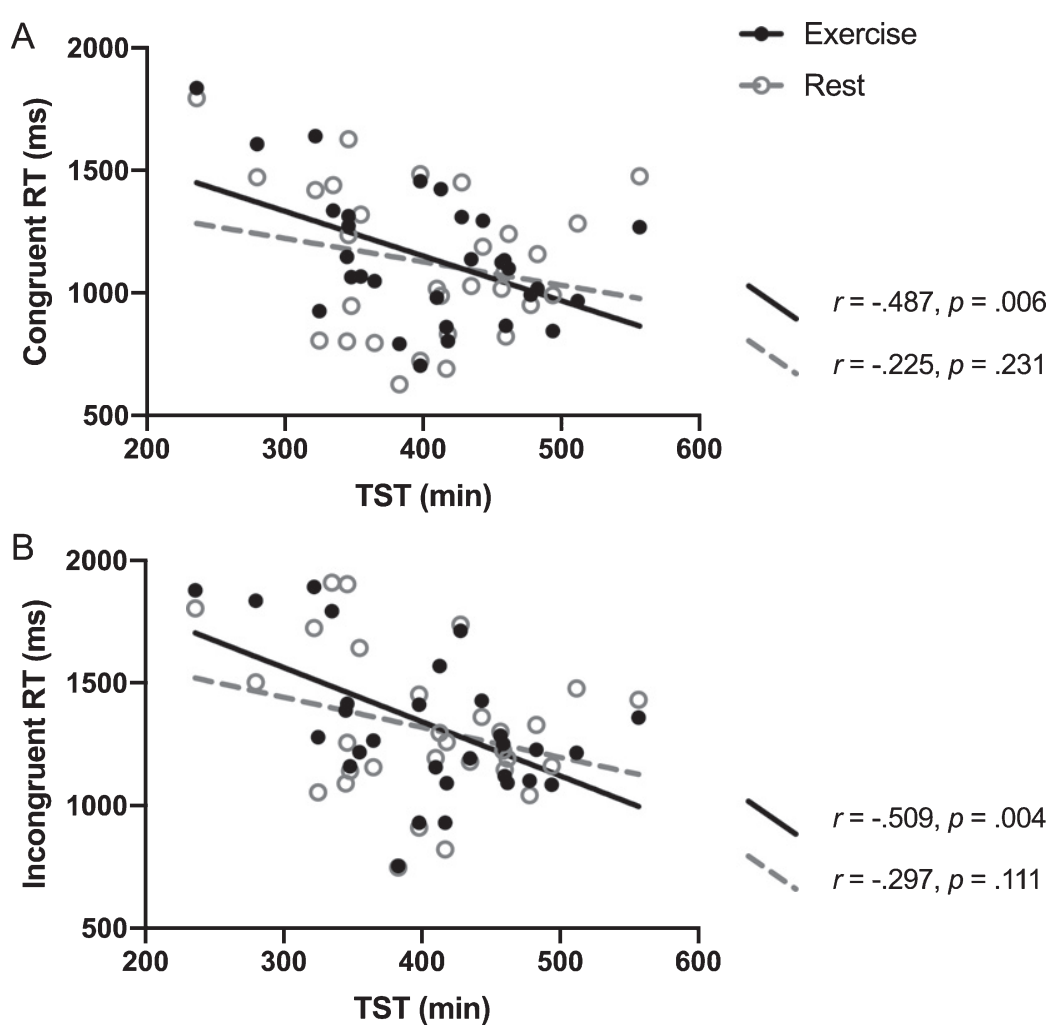

Fig. 1. The correaltion between TST and the Stroop RT for exercise and rest conditions. Pearson's correlation $r$ and $p$-values indicate correaltion of TST and Stroop RT for each condition. Interactions were significant for both (A) and (B), with $p$-values of 0.029 and 0.022 , respectively.

the exercise and rest conditions were $136.1 \pm 18.9$ bpm and $66.9 \pm 8.7 \mathrm{bpm}$, respectively $(\mathrm{t}(29)=18.04$, $p<0.0001, d=4.70$ ), suggesting the exercise intervention produced the desired effect. Similarly, RPE during exercise $(14.3 \pm 1.2)$ was significantly greater relative to rest $(6.1 \pm 0.3 ; \mathrm{t}(29)=37.16, p<0.0001$, $d=9.38$ ). The mean RPE during exercise corresponded to a rating between the verbal anchors "Somewhat Hard" and "Hard", indicating that participants subjectively perceived the exercise at a moderate intensity as intended. None of the Stroop test performance outcomes demonstrated a significant main effect of exercise (Table 3 ).

\section{Sleep and exercise interactive effects}

Because there was a strong and significant correlation between WASO and SE $(r=-0.960, p<0.0001)$, we decided to include only one of these variables to avoid issues of multicollinearity. We selected WASO as an index of sleep fragmentation because it is not as dependent on the participant providing (errorprone) input on time into bed, which is required to compute SE. The multiple linear regression analysis revealed significant interactions between TST and conditions on RT during both Stroop congruent (interaction $b=-0.872,95 \% \mathrm{CI}=-1.605$ to $-0.139, p=0.029)$ and incongruent trials (interaction $b=-0.883,95 \% \mathrm{CI}=-1.385$ to $-0.110, p=0.022$ ) (see Fig. 1). More specifically, shorter TST was associated with longer Stroop congruent RT (and longer TST associated with shorter RT) after exercise ( $r=-0.487, p=0.006$ ) but there was no significant relationship between TST and congruent RT after the rest condition $(r=-0.225, p=0.231$; Fig. 1 , Panel A). Similarly, the correlation between TST and the Stroop incongruent RT after exercise was statistically significant $(r=-0.509, p=0.004)$, but no significant association was found after rest $(r=-0.297, p=0.111$; Fig. 1, Panel B). The interactions between WASO with conditions approached but did not reach significance for congruent RT $(b=2.279,95 \% \mathrm{CI}=-0.046$ to $4.603, p=0.055)$ or incongruent RT $(b=1.792,95 \% \mathrm{CI}=-0.291$ to 3.874 , $p=0.092$ ). 


\section{Mediation analyses}

\section{Preparatory step for mediation analysis}

TST and WASO were used as sleep variables for the mediation analysis. First, we examined the association between sleep and other factors that could potentially explain variance during the analysis. Since age was negatively correlated with TST $(r=-0.491, p=0.006)$, age was included as a covariate when testing TST as an independent variable. Age was not significantly associated with WASO ( $r=0.045, p=0.814)$, and thus, age was not included as a covariate when WASO was the independent variable.

\section{Mediation analysis step 1: Association between sleep and Stroop task performance}

First, significant indirect mediating pathway between sleep (IV) and Stroop task performance (DV) must be established before testing the direct effects of mediation. After covariate adjustment, we detected that TST was significantly correlated with exercise-induced changes in Stroop incongruent RT $(b=-0.441, \mathrm{t}(28)=-2.597, p=0.015)$. Conversely, this effect was not observed in the congruent RT $(b=-0.339, \mathrm{t}(28)=-1.909, p=0.067)$, interference score $(b=0.041, \mathrm{t}(28)=0.218, p=0.829)$, incongruent accuracy $(b=-0.043, \mathrm{t}(28)=-0.228$, $p=0.822)$, and congruent accuracy $(b=0.137$, $\mathrm{t}(28)=0.732, \quad p=0.470)$. We failed to detect a significant correlation between WASO and Stroop performance, including incongruent RT $(b=0.268, \mathrm{t}(28)=1.470, p=0.153)$, congruent $\mathrm{RT}$ $(b=0.293, \mathrm{t}(28)=1.623, p=0.116)$, interference score $(b=-0.182, \mathrm{t}(28)=-0.982, p=0.334)$, incongruent accuracy $(b=0.183, \mathrm{t}(28)=0.987, p=0.332)$, and congruent accuracy $(b=0.009, \mathrm{t}(28)=0.048$, $p=0.962$ ).

\section{Mediation analysis step 2: Association between sleep and $B G$ volume}

After establishing the indirect effects, the direct pathway must be established between the IV and mediator. Therefore, we identified the associations between sleep and the volumetric measures of the subcortical nuclei that compose the BG. Significant positive associations between TST and the volume of caudate $(b=0.400, \mathrm{t}(28)=2.310$, $p=0.028)$ and putamen $(b=0.372, \mathrm{t}(28)=2.120$, $p=0.043$ ) were observed, while these correlations were not significant in the globus pallidus $(b=0.023$, $\mathrm{t}(28)=0.124, p=0.903)$ or total GM $(b=-0.051$, $\mathrm{t}(28)=-0.270, p=0.789)$ volume. WASO was not significantly correlated with any of the subcomponents of the BG, including the caudate $(b=-0.136$, $\mathrm{t}(28)=-0.728, \quad p=0.473), \quad$ putamen $(b=-0.178$, $\mathrm{t}(28)=-0.959, p=0.346)$, globus pallidus $(b=0.027$, $\mathrm{t}(28)=0.142, p=0.888)$, or total $\mathrm{GM}$ volume $(b=-0.028, \mathrm{t}(28)=-0.149, p=0.883)$.

\section{Mediation analysis step 3: Association between Stroop task performance and BG volume}

The direct pathway between Stroop task performance (DV) and BG volume (M) must be established in the mediation model [40] in order to examine possible indirect mediating pathways between sleep and Stroop task performance through BG volume. Significant associations between the caudate volume and exercise-induced changes in the Stroop incongruent RT $(b=-0.528, \mathrm{t}(28)=-3.287, p=0.003)$ and congruent RT $(b=-0.444, \mathrm{t}(28)=-2.621$, $p=0.014)$ were observed. However, interference score $(b=0.139, \mathrm{t}(28)=0.744, p=0.463)$, incongruent accuracy $(b=0.071, \mathrm{t}(28)=0.379, p=0.707)$, and congruent accuracy $(b=-0.345, \mathrm{t}(28)=-1.943$, $p=0.062$ ) were not significantly associated with caudate volume. The volume of the putamen was not significantly correlated with incongruent RT $(b=-0.077, \mathrm{t}(28)=-0.406, p=0.688)$, congruent RT $(b=-0.023, \mathrm{t}(28)=-0.120, p=0.906)$, interference score $(b=-0.061, \mathrm{t}(28)=-0.321, p=0.751)$, incongruent accuracy $(b=-0.051, \mathrm{t}(28)=-0.271$, $p=0.789)$, or congruent accuracy $(b=-0.096$, $\mathrm{t}(28)=-0.509, p=0.615)$. We also failed to detect any significant associations between the globus pallidus and the incongruent RT $(b=-0.062$, $\mathrm{t}(28)=-0.328, p=0.745)$, congruent RT $(b=-0.106$, $\mathrm{t}(28)=-0.564, \quad p=0.577), \quad$ interference score $(b=0.063, \quad \mathrm{t}(28)=0.336, \quad p=0.739)$, incongruent accuracy $(b=-0.020, \mathrm{t}(28)=-0.106, p=0.917)$, and congruent accuracy $(b=-0.135, \mathrm{t}(28)=-0.721$, $p=0.477$ ).

\section{Mediation analysis step 4: Mediating pathways}

The presence of significant relationships between TST and caudate volume, and caudate volume and incongruent Stroop RT, suggest that only caudate volume met the assumptions for a mediation analysis. The mediation analysis was tested using the bootstrapping method with bias-corrected confidence estimates [39, 40] and included age as a covariate in the regression model. Results of the mediation analysis revealed a mediating role of the bilateral caudate volume in the relationship between 
A

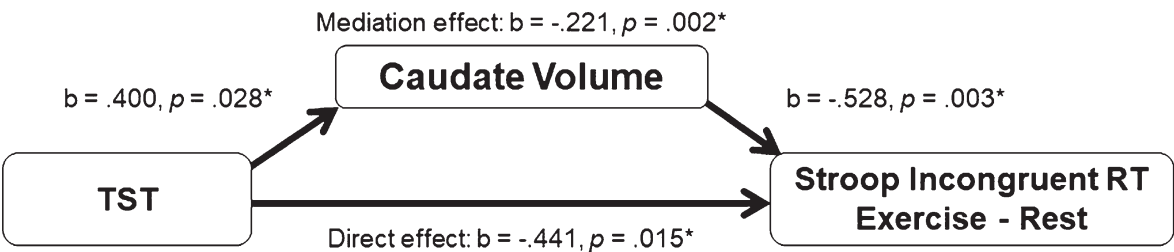

Indirect effect: $b=-.348, p=.085$

B

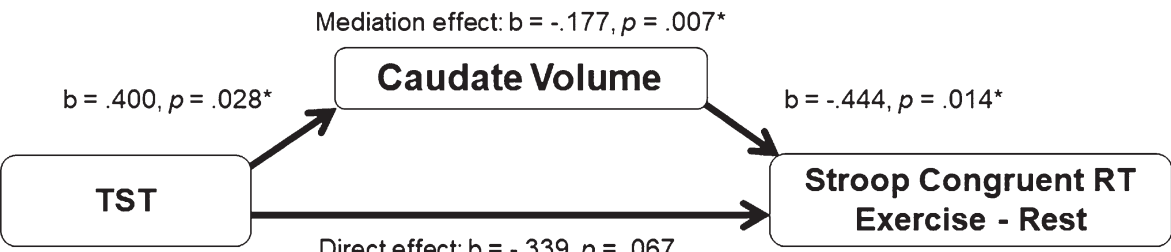

Indirect effect: $b=-.214, p=.225$

C

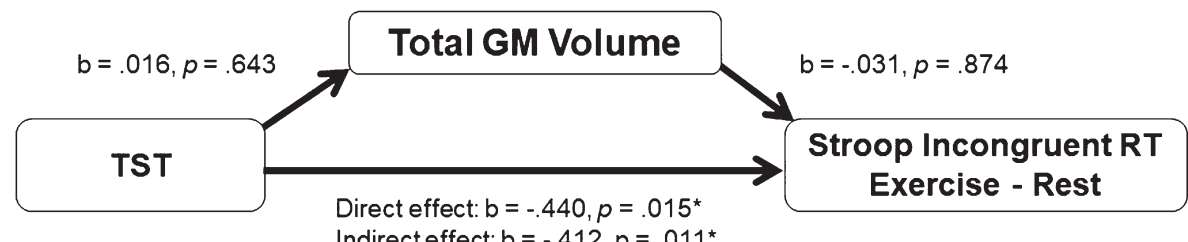

Fig. 2. (A) represents the indirect mediating pathway between TST and exercise-altered Stroop incongruent RT through the bilateral caudate volume. Mediation analysis of the relationship between TST and exercise-altered Stroop congruent RT with the bilateral caudate volume as a mediator is presented in (B). Mediation analysis of the relationship between TST and exercise-altered Stroop incongruent RT with the total gray matter as a mediator is illustrated in (C).

TST and exercise-altered Stroop incongruent RT (mediation coefficient $=-0.211,95 \% \mathrm{CI}=-0.818$ to $-0.035, p=0.002$ ). Additionally, the direct effect of TST on the Stroop incongruent RT change score was no longer significant $(b=-0.348, \mathrm{t}(28)=-1.788$, $p=0.085)$ when controlling for bilateral caudate volume; therefore, suggesting a mediation effect. Regarding Stroop congruent trials, the mediating effect of the bilateral caudate volume in the relationship between TST and exercise-altered RT was also significant (mediation coefficient $=-0.177,95 \%$ $\mathrm{CI}=-0.827$ to $-0.020, p=0.007$ ). However, since the indirect effect of TST on the Stroop congruent RT change was not significant $(b=-0.339$, $\mathrm{t}(28)=-1.909, p=0.067)$, this does not qualify as full mediation. In order to rule out the possibility that the mediation effect was due to a generalized effect of cortical tissue volume, we also tested total GM volume as a control. As shown in Fig. 2, total GM volume was not related to TST or Stroop performance, and thus by definition was not a mediator of the relationship between TST and Stroop performance.
Only bilateral caudate volume was shown to mediate the relationship between TST and exercise-induced changes in Stroop incongruent RT performance.

\section{DISCUSSION}

In the present study, we investigated the interaction of sleep and acute exercise on executive function performance, as well as the mediating role of BG volume in these associations. Our study has two major findings. First, greater sleep duration was associated with faster Stroop RT after exercise. Specifically, we observed an exercise-induced decrease in RT among those with longer TST and an exerciseinduced increase in RT among those with shorter TST. Second, the association between sleep duration and exercise-induced changes in executive function performance were mediated by the volume of the caudate nuclei, such that longer TST was correlated with shorter Stroop incongruent RT after exercise through greater caudate volume. 
Studies that have measured Stroop task performance after a single exercise session among older adults have reported mixed results. While significantly shorter Stroop RT has been found immediately after acute exercise [41], acute exercise does not appear to enhance Stroop task performance at multiple time points shortly after exercise (i.e., 5, 10, 15, and $120 \mathrm{~min}$ after exercise) [42]. Acute exercise promotes transient increases in neurotransmitters, neuromodulators, and neurotrophins, with subsequent expression of neural growth factors $[43,44]$. These neurophysiological alterations elicited by acute exercise are associated with enhanced prefrontal-dependent cognitive functioning [45]. Here, we extend this line of evidence by suggesting sleep duration potentially plays a significant role in the longer-lasting effects of acute exercise on executive function.

Although a main effect of exercise on Stroop task performance was not observed 1.5 hours after exercise cessation, we found that changes in Stroop RT after exercise were dependent on sleep duration. Individuals with longer TST had shorter RT after exercise, whereas those with shorter TST showed longer RT after exercise for both congruent and incongruent trials (Fig. 1). This pattern of results suggests that exercise does not improve executive function performance in those with short habitual sleep. Prior studies have observed that a shorter sleep duration was linked to increased perceived stress [46], impaired concentration, and decreased ability to accomplish daily tasks [47]. Psychobiological studies have suggested that inadequate TST engenders chronically high cortisol levels [48] and is associated with greater amount of perceived stress response after acute cognitive challenge via the Stroop test [49]. Based on these findings, we surmise that the physical strain associated with a single exercise bout might have acted as an extra stressor for shorter sleepers through stimulation of the stress-response system that possibly resulted in impaired executive function performance. Although moderate-intensity exercise can be expected to engage the hypothalamic-pituitaryadrenal axis [50], as well as the sympathetic nervous system [51], this interpretation warrants caution as we did not measure stress hormones in our study. One may also wonder if these effects would be similar or exacerbated among those with more severely disturbed sleep. Our participants did not report a history of problems initiating sleep or staying asleep whose TST $(432.2 \pm 80.4 \mathrm{~min})$ were in the range of recommended sleep duration for older adults (420-480 min)
[52], and were considered at low risk of obstructive sleep apnea (STOP-BANG score of $1.6 \pm 0.7$ ). Hence, our study supports previous research demonstrating that TST is associated with better executive function in healthy older adults $[53,54]$, but may not extend to those diagnosed with a sleep disorder.

In contrast to the associations with shorter TST, for those with longer TST, our findings suggest performing an acute bout of exercise may have elicited cognitive improvements that were sustained for at least 90 minutes. Based on the linear regression equations calculated for Fig. 1, the point of intersection between the exercise and rest conditions was at 422 minutes of TST for the incongruent RT variable, and 427 minutes for the congruent RT variable. This is consistent with research that suggests impaired cognitive function may occur for those who report less than 7 hours of sleep per night [55]. However, others have shown that cortical thinning is accelerated over time in those who self-report sleeping more and less than 7 hours per night, compared to who sleep 7 hours per night, suggesting a u-shaped function of TST [56]. We performed a post-hoc analysis to test the polynomial hypothesis, but did not find a significant quadratic function of TST on Stroop performance in the exercise or rest conditions.

\section{Mediation by caudate volume}

We found that the volume of the caudate was a significant mediator of the relationship between TST and incongruent trial RT difference between exercise and rest (Figure 2; Panel A). Importantly, caudate volume accounted for the relationship between TST and exercise-related performance in executive function. In other words, the effect of exercise to improve Stroop response time in those with longer TST was explained by their greater caudate volume. Previous studies have suggested that caudate volume is a significant mediator between cardiorespiratory fitness and task-switching performance [22] and that greater cardiorespiratory fitness is associated with better executive function performance and greater BG volume [23]. Although there are many differences between these earlier studies and our study, including the study design (cross-sectional vs. quasi-experimental within-subject design), selection of cognitive test (Flanker, Task-switch vs. Stroop) and variables of interest (cardiorespiratory fitness vs. TST and acute exercise), these results collectively point out the important role of the caudate nuclei 
in the relationship between executive function and exercise. Therefore, the present study complements these earlier findings by shedding light on the mediating role of the caudate in its relation to sleep and acute exercise related improvements in executive function.

The question remains as to why the significant mediating effects appeared only in the incongruent Stroop trials. The incongruent condition (e.g. the word blue presented in color green) necessitates inhibition of the innate response and filtering conflicting information between word color and meaning. This requires suppressing task-irrelevant information, eliciting a higher demand of preparation, initiation of cognitive process, and motor responses [57]. A previous study observed greater functional MRI activation in the left caudate during incongruent Stroop trials only, but not during congruent or neutral trials, highlighting the role of the caudate in suppressing and controlling task-irrelevant information during completion of tasks with a higher-order executive function [58]. Our finding for a mediation effect only for incongruent trial performance is in agreement with this earlier finding.

Nevertheless, the direct association between TST and congruent trial RT fell just short of being statistically significant, though the effects were in the same direction (Fig. 2; Panel B). Although not meeting criteria for full mediation, caudate volume demonstrated a similar mediation pattern for the relationship between TST and the Stroop congruent trials. Given the similar pattern of results, it is quite possible these results for the congruent trials reflect a Type II error, by accepting the null hypothesis when it may be false. On the other hand, the stronger effects for the incongruent condition suggest that the impact of TST and its mediation by caudate volume may not manifest until the executive control network is sufficiently challenged. This similar pattern for both in the congruent and incongruent trials certainly explain the absence of the mediating effect for the exercise-related changes in Stroop interference score (incongruent minus congruent), which indexes the additional time needed to respond to the incongruent relative to the congruent trials. The consistent direction of the effects for both types of Stroop trials suggests a general involvement of the caudate nuclei during the Stroop task, but is particularly strong when greater interference is present. This interpretation is consistent with the role of the frontal-striatal projections serving as a gain modulator that increases the signal to noise ratio with these networks [19].
Lastly, the fact that total GM volume did not show statistical significance in the mediation model implies the mediation effect may be specific to the caudate, and not associated with total brain volume (Fig. 2; Panel C).

\section{Potential mechanisms}

Although the precise neurophysiological mechanism that is driving the mediation effect we observed is not entirely clear, frontal-striatal dopaminergic projections certainly are a possible candidate for the underlying mechanism of the mediating effects we observed. The caudate engages in executive function with its connection to medial, ventral, and dorsolateral prefrontal cortex through the frontalstriatal pathway [59]. These projections impact frontal networks involved in the cognitive control of attention, response inhibition, and working memory [60]. Indeed, deficits in dopamine D2/D3 receptor availability in the caudate are related to impaired prefrontal cortex function and executive dysfunction [61]. Given that global brain volume [62], including the caudate [63], decreases in old age, and short sleep duration is associated with greater age-related brain atrophy, it is plausible that shorter TST may accelerate age-related caudate volume loss. It follows that caudate volume loss presumably results in less dopamine receptor availability, ultimately impairing the executive function performance.

Our assumption regarding the mediating effects through caudate dopaminergic projections is also supported by exercise studies. Dopamine is the reward neurotransmitter and dopaminergic neurocircuitry is involved in reward process where the brain triggers its release after accomplishment of task [64]. It is well-established in rodent studies that the nucleus accumbens, a major component of the ventral striatum situated between the caudate and putamen, mediates the dopamine receptor pathways and rewarding aspect of exercise $[65,66]$. Exercise results in increased dopaminergic activity in the BG and these ascending dopaminergic projections have shown to increase availability of dopaminergic neurotransmission [67, 68], which is associated with cognitive facilitation. Thus, the present data are consistent with these evidence by showing dopaminergic neurocircuitry in the caudate nuclei is possibly associated with alteration in Stroop task performance after a single session of exercise. 


\section{Limitations and future directions}

There are several limitations in the present study. Firstly, since our participants regularly participated in exercise ( $\geq 3$ days/week), were highly educated (93\% had college or graduate degree), did not have a diagnosis of cardiovascular disease or mental disorder (e.g. hypertension, diabetes, or depression), were at low-risk of sleep apnea, and were predominantly Caucasian $(76 \%)$, our results may not generalize to other samples. Future studies need to investigate whether these effects are replicable in clinical populations (e.g. chronic sleep disturbance, mild cognitive impairment or Alzheimer's disease) to understand if addressing both sleep and exercise can help preserve cognitive function beyond addressing only one or the other. Second, there was an approximately 90-min interval between the cessation of acute exercise and the Stroop task. This was because we had a series of MRI scans that lasted approximately 90 minutes after each of the experimental sessions prior to performance of the Stroop task. The Stroop task was included after the MRI session based on work that has shown the effects of acute exercise on executive function may last up to 2 hours after exercise [69]. Future studies should assess the immediate and shorter term effects of exercise and its interaction with sleep by administering cognitive tasks more closely following exercise. Because we were testing a priori hypotheses for the interaction effects, the present study did not adjust for multiple comparisons; nevertheless, these findings should be interpreted cautiously until they are replicated.

\section{CONCLUSION}

Our findings suggest sufficient total sleep time may be an important modifier of the effects of acute exercise on cognition. Moreover, the association between longer sleep duration and exercise-induced improvement in executive function performance may be mediated through greater volume of the caudate. The present findings suggest that consideration of the interactive effects between lifestyle factors, such as physical activity and sleep behavior, may be an effective strategy to promote healthy cognitive aging. Considering previous findings that chronic exercise has marked effects on disrupted sleep [70-72], the association between long-term exercise-induced alterations in sleep and cognitive function, including the possible mediating role of the BG, should be examined in the future.

\section{ACKNOWLEDGEMENTS}

We thank the participants for their time and effort while participating in this study. Support for this study was provided by the Department of Kinesiology and School of Public Health at the University of Maryland. Lauren R. Weiss was supported by NSF Award DGE-1632976.

\section{CONFLICT OF INTEREST}

Dr. Adam Spira received an honorarium from Springer Nature Switzerland AG for Guest Editing a Special Issue of Current Sleep Medicine Reports. None of the other authors have conflict of interest to report.

\section{REFERENCES}

[1] Prinz PN, Vitiello MV, Raskind MA, Thorpy MJ. Sleep disorders and aging. N Engl J Med. 1990;323(8):520-526.

[2] Rediehs MH, Reis JS, Creason NS. Sleep in old age: Focus on gender differences. Sleep. 1990;13(5):410-424.

[3] Bliwise DL, Foley DJ, Vitiello MV, Ansari FP, Ancoli-Israel S, Walsh JK. Nocturia and disturbed sleep in the elderly. Sleep Med. 2009;10(5):540-548.

[4] Foley D, Ancoli-Israel S, Britz P, Walsh J. Sleep disturbances and chronic disease in older adults: Results of the 2003 National Sleep Foundation Sleep in America Survey. J Psychosom Res. 2004;56(5):497-502.

[5] Yaffe K, Falvey CM, Hoang T. Connections between sleep and cognition in older adults. Lancet Neurol. 2014;13(10):1017-1028

[6] Altena E, Vrenken H, Van Der Werf YD, van den Heuvel OA, Van Someren EJ. Reduced orbitofrontal and parietal gray matter in chronic insomnia: A voxel-based morphometric study. Biol Psychiatry. 2010;67(2):182-185.

[7] Lim AS, Kowgier M, Yu L, Buchman AS, Bennett DA. Sleep fragmentation and the risk of incident Alzheimer's disease and cognitive decline in older persons. Sleep. 2013;36(7):1027-1032.

[8] Blackwell T, Yaffe K, Ancoli-Israel S, Redline S, Ensrud KE, Stefanick ML, et al. Association of sleep characteristics and cognition in older community-dwelling men: The MrOS sleep study. Sleep. 2011;34(10):1347-1356.

[9] Oosterman JM, Van Someren EJ, Vogels RL, Van Harten B, Scherder EJ. Fragmentation of the rest-activity rhythm correlates with age-related cognitive deficits. J Sleep Res. 2009;18(1):129-135.

[10] Bastien CH, Fortier-Brochu E, Rioux I, LeBlanc M, Daley $\mathrm{M}$, Morin CM. Cognitive performance and sleep quality in the elderly suffering from chronic insomnia: Relationship between objective and subjective measures. J Psychosom Res. 2003;54(1):39-49.

[11] Nebes RD, Buysse DJ, Halligan EM, Houck PR, Monk TH. Self-reported sleep quality predicts poor cognitive performance in healthy older adults. J Gerontol Ser B. 2009;64(2):180-187.

[12] Angevaren M, Aufdemkampe G, Verhaar HJ, Aleman A, Vanhees L. Physical activity and enhanced fitness 
to improve cognitive function in older people without known cognitive impairment. Cochrane Database Syst Rev. 2008;3(3):1-73.

[13] Kamijo K, Hayashi Y, Sakai T, Yahiro T, Tanaka K, Nishihira Y. Acute effects of aerobic exercise on cognitive function in older adults. J Gerontol Ser B. 2009;64(3):356363.

[14] Eriksen BA, Eriksen CW. Effects of noise letters upon the identification of a target letter in a nonsearch task. Percept Psychophys. 1974;16(1):143-149.

[15] Stroop JR. Studies of interference in serial verbal reactions. J Exp Psychol. 1935;18(6):643.

[16] Chang Y-K, Chu C-H, Wang C-C, Wang Y-C, Song T-F, Tsai C-L, et al. Dose-response relation between exercise duration and cognition. Med Sci Sports Exerc. 2015;47(1): 159-165.

[17] Vetrivelan R, Qiu M-H, Chang C, Lu J. Role of basal ganglia in sleep-wake regulation: Neural circuitry and clinical significance. Front Neuroanat. 2010;4:145.

[18] Lazarus M, Chen J-F, Urade Y, Huang Z-L. Role of the basal ganglia in the control of sleep and wakefulness. Curr Opin Neurobiol. 2013;23(5):780-785.

[19] Crosson B, Benjamin M, Levy I. Role of the basal ganglia in language and semantics: Supporting cast. Cambridge University Press: New York, NY, USA; 2007. 219-243 p.

[20] Fisher BE, Petzinger GM, Nixon K, Hogg E, Bremmer $\mathrm{S}$, Meshul CK, et al. Exercise-induced behavioral recovery and neuroplasticity in the 1-methyl-4-phenyl-1, 2, 3, 6-tetrahydropyridine-lesioned mouse basal ganglia. J Neurosci Res. 2004;77(3):378-390.

[21] Petzinger GM, Walsh JP, Akopian G, Hogg E, Abernathy A, Arevalo P, et al. Effects of treadmill exercise on dopaminergic transmission in the 1-methyl-4-phenyl-1, 2, 3, 6-tetrahydropyridine-lesioned mouse model of basal ganglia injury. J Neurosci. 2007;27(20):5291-5300.

[22] Verstynen TD, Lynch B, Miller DL, Voss MW, Prakash RS, Chaddock L, et al. Caudate nucleus volume mediates the link between cardiorespiratory fitness and cognitive flexibility in older adults. J Aging Res. 2012;2012.

[23] Chaddock L, Erickson KI, Prakash RS, VanPatter M, Voss MW, Pontifex MB, et al. Basal ganglia volume is associated with aerobic fitness in preadolescent children. Dev Neurosci. 2010;32(3):249-256.

[24] Chung F, Abdullah HR, Liao P. STOP-Bang questionnaire: A practical approach to screen for obstructive sleep apnea. Chest. 2016;149(3):631-638.

[25] Blair SN, Haskell WL, Ho P, Paffenbarger RS, Vranizan KM, Farquhar JW, et al. Assessment of habitual physical activity by a sevenday recall in a community survey and controlled experiments. Am J Epidemiol. 1985;122(5):794804.

[26] Folstein MF, Folstein SE, McHugh PR. "Mini-mental state": A practical method for grading the cognitive state of patients for the clinician. J Psychiatr Res. 1975;12(3): 189-198.

[27] Won J, Alfini AJ, Weiss LR, Michelson CS, Callow DD, Ranadive SM, et al. Semantic Memory Activation After Acute Exercise in Healthy Older Adults. J Int Neuropsychol Soc. 2019;1-12.

[28] Sadeh A, Acebo C. The role of actigraphy in sleep medicine. Sleep Med Rev. 2002;6(2):113-124.

[29] Blackwell T, Redline S, Ancoli-Israel S, Schneider JL, Surovec S, Johnson NL, et al. Comparison of sleep parameters from actigraphy and polysomnography in older women: The SOF study. Sleep. 2008;31(2):283-291.
[30] Jean-Louis G, Kripke DF, Mason WJ, Elliott JA, Youngstedt SD. Sleep estimation from wrist movement quantified by different actigraphic modalities. J Neurosci Methods. 2001;105(2):185-191.

[31] Cole RJ, Kripke DF, Gruen W, Mullaney DJ, Gillin JC. Automatic sleep/wake identification from wrist activity. Sleep. 1992;15(5):461-469.

[32] Borg G. Perceived exertion as an indicator of somatic stress. Scand J Rehabil Med. 1970;2(2):92.

[33] Cox RW. AFNI: Software for analysis and visualization of functional magnetic resonance neuroimages. Comput Biomed Res. 1996;29(3):162-173.

[34] Fischl B. FreeSurfer. Neuroimage. 2012;62(2):774-781.

[35] Reuter M, Schmansky NJ, Rosas HD, Fischl B. Withinsubject template estimation for unbiased longitudinal image analysis. Neuroimage. 2012;61(4):1402-1418.

[36] Raz N, Lindenberger U, Rodrigue KM, Kennedy KM, Head $\mathrm{D}$, Williamson $\mathrm{A}$, et al. Regional brain changes in aging healthy adults: General trends, individual differences and modifiers. Cereb Cortex. 2005;15(11):1676-1689.

[37] Scahill RI, Frost C, Jenkins R, Whitwell JL, Rossor MN, Fox NC. A longitudinal study of brain volume changes in normal aging using serial registered magnetic resonance imaging. Arch Neurol. 2003;60(7):989-994.

[38] Buckner RL. Memory and executive function in aging and AD: Multiple factors that cause decline and reserve factors that compensate. Neuron. 2004;44(1):195-208.

[39] Hayes AF. PROCESS: A versatile computational tool for observed variable mediation, moderation, and conditional process modeling. University of Kansas, KS; 2012.

[40] Preacher KJ, Hayes AF. Asymptotic and resampling strategies for assessing and comparing indirect effects in multiple mediator models. Behav Res Methods. 2008;40(3): 879-891.

[41] Chang Y-K, Etnier JL. Effects of an acute bout of localized resistance exercise on cognitive performance in middleaged adults: A randomized controlled trial study. Psychol Sport Exerc. 2009;10(1):19-24.

[42] Barella LA, Etnier JL, Chang Y-K. The immediate and delayed effects of an acute bout of exercise on cognitive performance of healthy older adults. J Aging Phys Act. 2010;18(1):87-98.

[43] Ferris LT, Williams JS, Shen C-L. The effect of acute exercise on serum brain-derived neurotrophic factor levels and cognitive function. Med Sci Sports Exerc. 2007;39(4):728734.

[44] Van Praag H, Shubert T, Zhao C, Gage FH. Exercise enhances learning and hippocampal neurogenesis in aged mice. J Neurosci. 2005;25(38):8680-8685.

[45] Hwang J, Brothers RM, Castelli DM, Glowacki EM, Chen YT, Salinas MM, et al. Acute high-intensity exercise-induced cognitive enhancement and brain-derived neurotrophic factor in young, healthy adults. Neurosci Lett. 2016;630:247-253.

[46] Choi D-W, Chun S-Y, Lee SA, Han K-T, Park E-C. Association between Sleep Duration and Perceived Stress: Salaried Worker in Circumstances of High Workload. Int J Environ Res Public Health. 2018;15(4):796.

[47] Roth T, Ancoli-Israel S. Daytime consequences and correlates of insomnia in the United States: Results of the 1991 National Sleep Foundation Survey. II. Sleep J Sleep Res Sleep Med. 1999;

[48] Leproult R, Copinschi G, Buxton O, Van Cauter E. Sleep loss results in an elevation of cortisol levels the next evening. Sleep. 1997;20(10):865-870. 
[49] Okamura H, Tsuda A, Yajima J, Mark H, Horiuchi S, Toyoshima N, et al. Short sleeping time and psychobiological responses to acute stress. Int J Psychophysiol. 2010;78(3):209-214.

[50] Stranahan AM, Lee K, Mattson MP. Central mechanisms of HPA axis regulation by voluntary exercise. Neuromolecular Med. 2008;10(2):118-127.

[51] Christensen NJ, Galbo H. Sympathetic nervous activity during exercise. Annu Rev Physiol. 1983;45(1):139-153.

[52] Hirshkowitz M, Whiton K, Albert SM, Alessi C, Bruni O, DonCarlos L, et al. National Sleep Foundation's sleep time duration recommendations: Methodology and results summary. Sleep Health. 2015;1(1):40-43.

[53] Lo JC, Groeger JA, Cheng GH, Dijk D-J, Chee MW. Selfreported sleep duration and cognitive performance in older adults: A systematic review and meta-analysis. Sleep Med. 2016;17:87-98.

[54] Lo JC, Loh KK, Zheng H, Sim SK, Chee MW. Sleep duration and age-related changes in brain structure and cognitive performance. Sleep. 2014;37(7):821-821.

[55] Devore EE, Grodstein F, Duffy JF, Stampfer MJ, Czeisler CA, Schernhammer ES. Sleep duration in midlife and later life in relation to cognition. J Am Geriatr Soc. 2014;62(6): 1073-1081.

[56] Spira AP, Gonzalez CE, Venkatraman VK, Wu MN, Pacheco J, Simonsick EM, et al. Sleep duration and subsequent cortical thinning in cognitively normal older adults. Sleep. 2016;39(5):1121-1128.

[57] Bugg JM, Jacoby LL, Toth JP. Multiple levels of control in the Stroop task. Mem Cognit. 2008;36(8):1484-1494.

[58] Ali N, Green DW, Kherif F, Devlin JT, Price CJ. The role of the left head of caudate in suppressing irrelevant words. J Cogn Neurosci. 2010;22(10):2369-2386.

[59] Lehéricy S, Ducros M, Van De Moortele P-F, Francois C, Thivard L, Poupon C, et al. Diffusion tensor fiber tracking shows distinct corticostriatal circuits in humans. Ann Neurol. 2004;55(4):522-529.

[60] Aron AR, Poldrack RA, Wise SP. Cognition: Basal ganglia role. 2009;

[61] Ballard ME, Dean AC, Mandelkern MA, London ED. Striatal dopamine D2/D3 receptor availability is associated with executive function in healthy controls but not methamphetamine users. PloS One. 2015;10(12):e0143510.

[62] Fotenos AF, Snyder AZ, Girton LE, Morris JC, Buckner RL. Normative estimates of cross-sectional and longitudinal brain volume decline in aging and AD. Neurology. 2005;64(6):1032-1039.

[63] Jernigan TL, Archibald SL, Fennema-Notestine C, Gamst AC, Stout JC, Bonner J, et al. Effects of age on tissues and regions of the cerebrum and cerebellum. Neurobiol Aging. 2001;22(4):581-594.

[64] Wise RA, Rompre P-P. Brain dopamine and reward. Annu Rev Psychol. 1989;40(1):191-225.

[65] Roberts MD, Gilpin L, Parker KE, Childs TE, Will MJ, Booth FW. Dopamine D1 receptor modulation in nucleus accumbens lowers voluntary wheel running in rats bred to run high distances. Physiol Behav. 2012;105(3):661-668.

[66] Greenwood BN, Foley TE, Le TV, Strong PV, Loughridge $\mathrm{AB}$, Day HE, et al. Long-term voluntary wheel running is rewarding and produces plasticity in the mesolimbic reward pathway. Behav Brain Res. 2011;217(2):354-362.

[67] Hattori S, Naoi M, Nishino H. Striatal dopamine turnover during treadmill running in the rat: Relation to the speed of running. Brain Res Bull. 1994;35(1):41-49.

[68] Petzinger GM, Fisher BE, Van Leeuwen J-E, Vukovic M, Akopian G, Meshul CK, et al. Enhancing neuroplasticity in the basal ganglia: The role of exercise in Parkinson's disease. Mov Disord. 2010;25(S1):S141-S145.

[69] Basso JC, Shang A, Elman M, Karmouta R, Suzuki WA. Acute exercise improves prefrontal cortex but not hippocampal function in healthy adults. J Int Neuropsychol Soc. 2015;21(10):791-801.

[70] King AC, Oman RF, Brassington GS, Bliwise DL, Haskell WL. Moderate-intensity exercise and self-rated quality of sleep in older adults: A randomized controlled trial. Jama. 1997;277(1):32-37.

[71] Kline CE, Crowley EP, Ewing GB, Burch JB, Blair SN, Durstine JL, et al. The effect of exercise training on obstructive sleep apnea and sleep quality: A randomized controlled trial. Sleep. 2011;34(12):1631-1640.

[72] Youngstedt SD. Effects of exercise on sleep. Clin Sports Med. 2005;24(2):355-365. 